\title{
Life-threatening acute myocardial infarction due to left main dissection during radiofrequency transcatheter ablation of atrial tachycardia
}

\author{
Alessandro Cafaro ${ }^{1}$, Annagrazia Cecere ${ }^{2}$, Roberta Ruggieri ${ }^{2}$, Elia Iorio ${ }^{1}$, \\ Francesco Giardinelli ${ }^{3}$, Marco Matteo Ciccone ${ }^{2}$, Vito Casamassima ${ }^{1}$, \\ Massimo Grimaldi ${ }^{1}$, Tommaso Langialonga ${ }^{1}$, Martino Pepe $^{2}$ \\ ${ }^{1}$ Department of Cardiology, General Hospital "F. Miulli”, Acquaviva delle Fonti, Bari, Italy \\ ${ }^{2}$ Cardiovascular Diseases Section, Department of Emergency and Organ Transplantation (DETO), \\ University of Bari, Bari, Italy \\ ${ }^{3}$ Department of Cardiology, Hospital "Santissima Annunziata”, Taranto, Italy
}

A 65-year-old woman was hospitalized for recurrent episodes of atrial tachycardia; echocardiography showed dilated left ventricle with depressed systolic function (ejection fraction 30\%), without significant epicardial coronary stenoses at angiography.

An electrophysiological study identified the origin of the arrhythmia in the posterior and midseptal walls of the right atrium. A radiofrequency transcatheter ablation (RTA) was performed through the right femoral vein approach: the right atrium was reached and a radiofrequency energy application of $35 \mathrm{~W}$ was delivered. During an inducibility test the patient complained of retrosternal pain associated with ST elevation in the V1 lead (Fig. 1A-D).

The subsequent emergency angiography showed ostial subocclusion of the left anterior descending (LAD) artery, suggesting a coronary dissection. At the second contrast injection the distal left main (LM) occluded. A percutaneous coronary intervention with a $3.5 \times 38 \mathrm{~mm}$ zotarolimus-eluting stent implantation at the LAD ostium was performed (Fig. 1E-J). After restoration of a Thrombolysis in Myocardial Infarction (TIMI) flow grade 3 in the LAD, an intravascular ultrasound (IVUS) run revealed a dissection flap at the level of the LM, managed with a second $4.5 \times 15 \mathrm{~mm}$ zotarolimus-eluting stent implantation. A good angiographic result was confirmed by a final IVUS run that demonstrated correct stent apposition through the LM-LAD axis (Fig. 1K-O). At 1 month follow-up patient's ejection fraction rose to $45 \%$.

According to available research, this is the only case in the literature of life-threatening LM dissection during right atrium RTA through a venous approach in which a fortuitous coronary ostia engagement can definitely be ruled out.

Conflict of interest: None declared

Address for correspondence: Dr. Annagrazia Cecere, Cardiovascular Diseases Section, Department of Emergency and Organ Transplantation (DETO), University of Bari, Italy, tel: +0039 080 5592996, e-mail: agcecere@gmail.com 


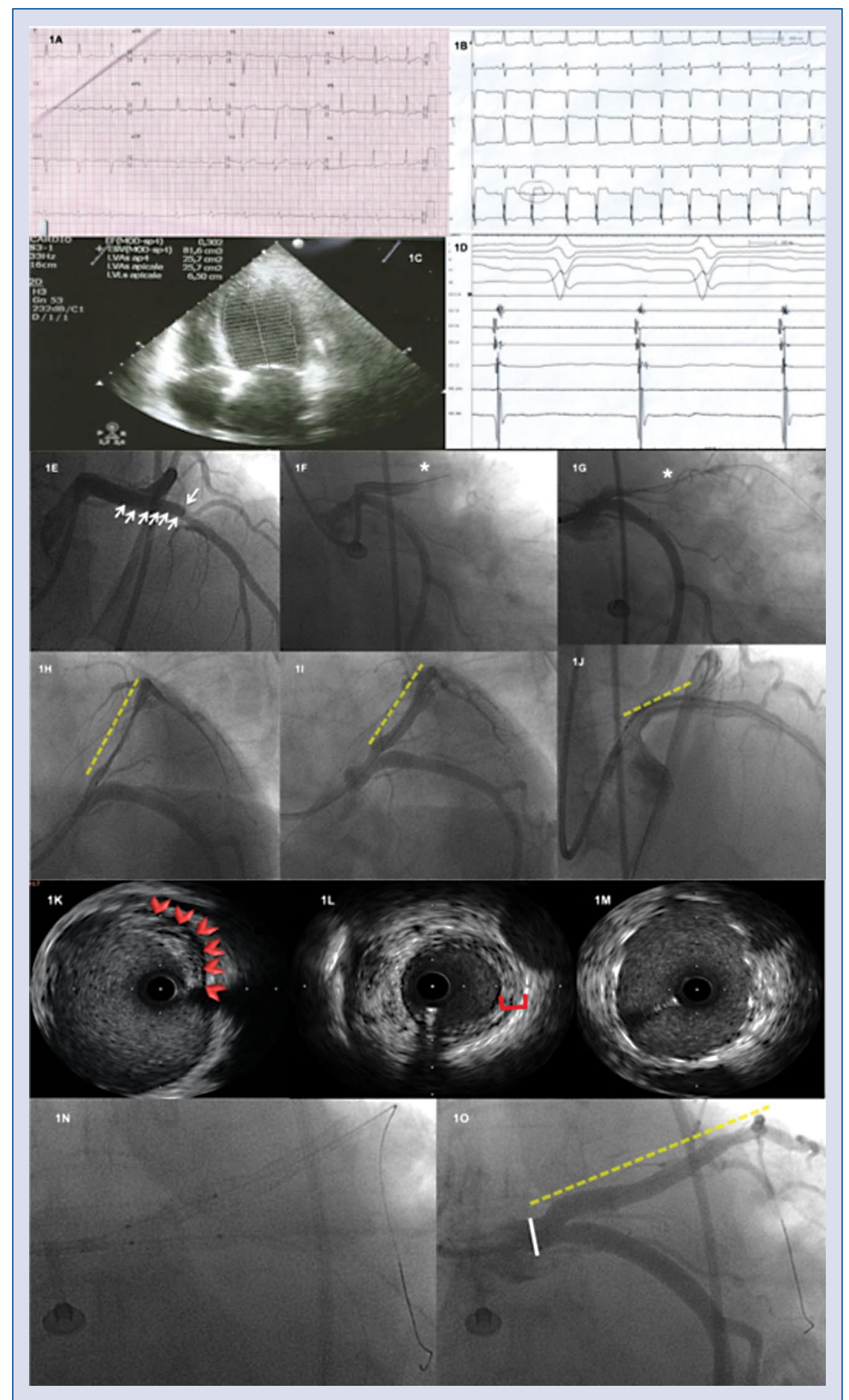

Figure 1. A. Normal electrocardiogram at admission; B. Electrocardiogram revealing ST elevation in V1; C. Transthoracic echocardiography at admission showing dilated left ventricle with $30 \%$ ejection fraction; D. Monitor polygraph record during transcatheter ablation; E. Left anterior descending coronary artery (LAD) dissection (white arrowheads); F, G. Abrupt total LAD closure (asterisks) and guidewire crossing; H, I. LAD drug eluting stent (DES) positioning and implantation (yellow dashed lines); J. DES positioning on left main (LM)-LAD (yellow dashed lines); K, L, M. Intravascular ultrasound (IVUS) run: IVUS images showing the extension of the dissection flap at the level of the LM (K: red arrowheads) and the optimal stent covering after stenting (M). IVUS at the level of the mid LAD shows medial thickening (L: between brackets); N: Final optimization with kissing balloon technique; O. Final angiographic result after LM-LAD percutaneous coronary intervention with DES implantation (yellow dashed lines). 